\title{
Detection of Voltage Sag using An Adaptive Extended Kalman Filter Based on Maximum Likelihood
}

\author{
Yanhui $\mathrm{Xi}^{\dagger}$, Zewen $\mathrm{Li}^{*}$, Xiangjun Zeng* and Xin Tang*
}

\begin{abstract}
An adaptive extended Kalman filter based on the maximum likelihood (EKF-ML) is proposed for detecting voltage sag in this paper. Considering that the choice of the process and measurement error covariance matrices affects seriously the performance of the extended Kalman filter (EKF), the EKF-ML method uses the maximum likelihood method to adaptively optimize the error covariance matrices and the initial conditions. This can ensure that the EKF has better accuracy and faster convergence for estimating the voltage amplitude (states). Moreover, without more complexity, the EKF-ML algorithm is almost as simple as the conventional EKF, but it has better anti-disturbance performance and more accuracy in detection of the voltage sag. More importantly, the EKF-ML algorithm is capable of accurately estimating the noise parameters and is robust against various noise levels. Simulation results show that the proposed method performs with a fast dynamic and tracking response, when voltage signals contain harmonics or a pulse and are jointly embedded in an unknown measurement noise.
\end{abstract}

Keywords: Voltage sag, Adaptive estimation, The extended Kalman filter, The maximum likelihood method, Wavelet transform

\section{Introduction}

Power quality (PQ) problems can be described as any disturbance in the electrical power supply such as voltage sag, swell and the presence of harmonics, which can result in failure or mal-operation of customer's equipment. Voltage sag, which refers to the reduction of RMS (root mean square) voltage, has become one of the most common power quality problems and accounts for the vast majority of problems experimented by end users. Thus, the fast detection and analysis of the voltage sag have been a major research concern of power engineers in recent years.

The voltage sag is characterized by their magnitude and duration. A wide variety of signal processing methods have been presented to detect the voltage sag, such as wavelet transform (WT) [1-2] and Kalman filter (KF) [3-4]. The WT technique provides a powerful tool for analyzing localized variations of power signals, and can be used to detect and localize voltage sag by decomposing a power signal into time-frequency space. Also, it can estimate the harmonic components in power system. Recently, Considering the effect of different combinations of disturbances, a hybrid discrete WT is proposed to detect the voltage sag in harmonics and flicker [5]. Although the WT method

$\dagger$ Corresponding Author: Hunan Province Higher Education Key Laboratory of Power System Safety Operation and Control (Changsha University of Science and Technology) China. (xiyanhui@126.com)

* Hunan Province Higher Education Key Laboratory of Power System Safety Operation and Control (Changsha University of Science and Technology) China. (1zw0917@163.com)

Received: August 4, 2016 ; Accepted: February 1, 2017 shows good results in detection of the voltage sag, it exhibits some disadvantages, such as complicated computation, sensitivity to noise level, and the dependency of its accuracy on the selection of mother wavelet. To overcome some of the above drawbacks, the S-transform (ST) uses a variant window length according to Fourier transform Kernel, which makes it obtain more accurate time resolution in high frequency parts and more frequency resolution in low frequency parts [6]. However, they also suffer from leakage effects and inaccuracies in the presence of noise with low signal-to-noise ratio (SNR). Moreover, all these time frequency-domain methods can't perform accurately for detection of sudden or fast changes in waveform, e.g. the power signal containing a pulse.

For accurate detection of the voltage sag, the Kalman filter approach based on time-domain, which is known to be simple and robust, attracts many attentions as it can estimate accurate amplitude, phase and frequency of the fundamental and harmonic components of a signal disturbed by white noise. However, the choice of the noise covariance matrices, which include the measurement noise covariance matrix $R$ and the process noise covariance matrix $Q$, is crucial in $\mathrm{KF}$ algorithm [7-9]. For the optimal choice of the noise covariance matrices, various improved $\mathrm{KF}$ algorithms have been applied to power system harmonic state estimation. For example, considering the uncertainty of the process noise covariance matrix, $\mathrm{Yu}[10]$ proposed an adaptive Kalman filter method in which two basic $Q$ models can be switched for steadystate and transient estimation. As a comparison, Shih and Huang [11] adjusted the measurement noise parameter $R$ 
instead of $Q$ to increase the robustness of the EKF method. For accurate analysis of harmonic content and fundamental frequency, Kennedy [12] employed an adaptive KF algorithm by adopting a methodical approach to choosing the noise covariance matrices $R$ and $Q$ simultaneously. Also, Zhang [13] developed an adaptive Kalman filter with inflatable noise variances, which can efficiently indentify and reduce the impact of incorrect system modeling and erroneous measurements. Recently, for the optimal choice of error covariance matrices $R$ and $Q$, Jatoth [14] proposed adaptive genetic algorithm-adaptive particle swarm optimization (GA-APSO) aided unscented Kalman filter to estimate harmonic components. All these research results show the above adaptive KF techniques are superior to the conventional KF algorithms, but most of these adaptive algorithms may require heavy computational outlay and can't provide good robustness with respect to various noise levels. Moreover, the practical unknown measurement noises will slow the rate of convergence and make the estimation results inaccurate or even diverging. Thus, the error covariance matrices $R$ and $Q$ must be estimated as unknown parameters in a practical situation.

In statistics, maximum likelihood (ML) estimation is a well-known method of estimating the parameters of a statistical model given data, which was analyzed and vastly popularized by Fisher [15]. In general, for a fixed set of data and underlying statistical model, the method of maximum likelihood selects the set of values of the model parameters that maximizes the likelihood function. Intuitively, this maximizes the "agreement" of the selected model with the observed data, and for discrete random variables it indeed maximizes the probability of the observed data under the resulting distribution, such as the normal (Gaussian) distribution. Maximum likelihood parameter estimation has been widely applied to many fields [16-17].

In this paper, a more robust and adaptive EKF algorithm is proposed for detection of voltage sag, which combines the EKF and the ML (EKF-ML). In the EKF-ML method, the maximum likelihood (ML) method is used to adaptively optimize the error covariance matrices $R, Q$ and the initial conditions as the parameters, and the EKF is used to estimate the voltage amplitude (states) simultaneously. Hence, the EKF-ML algorithm makes the observed results the most probable given the model and can obtain better performance relative to the conventional EKF (The conventional EKF assumed parameter values of the error covariance matrices as specified values). Another advantage of this algorithm is that it almost has the same computational complexity as the conventional EKF. Thus, the EKF-ML algorithm is simpler and less complex than other adaptive algorithms, such as the adaptive unscented Kalman filter algorithm [18], the unscented Kalman smoother algorithm [19] and adaptive particle swarm optimization (GA-APSO) aided unscented Kalman filter
[14]. All in all, the EKF-ML algorithm can accurately estimate the amplitudes of the voltage signals, and has fast response to the voltage sag even though the voltage signal is highly distorted by harmonics or a pulse and Gaussian noise with various SNR. What's more, the proposed adaptive EKF algorithm is capable of accurately estimating the noise parameters and is robust against various noise levels.

The main contribution of this paper is that the use of the maximum likelihood (ML) method to adaptively optimize the error covariance matrices $R, Q$ and the initial conditions as the parameters, which can make sure that the EKF has better accuracy and faster convergence.

The remainder of the paper is organized as follows. In Section 2, we propose the state space model of the power signal. Section 3 describes the EKF-ML method. Section 4 presents simulation studies of the EKF-ML, the conventional EKF and the WT. Some concluding remarks are contained in Section 5.

\section{The State Space Model of the Power Signal}

The discrete power signal is usually made up of a fundamental component plus some harmonic components and noise. Therefore, a typical power system observed signal can be expressed as

$$
y_{k}=A_{1, k} \cos \left(\omega k T_{s}+\varphi_{1}\right)+\sum_{r=2}^{M} A_{r, k} \cos \left(r \omega k T_{s}+\varphi_{r}\right)+v_{k}
$$

where $y_{k}$ is the observed signal, and $A_{1, k}, \omega, \varphi_{1}$ denote the amplitude, angular frequency and initial phase angle of the fundamental component, respectively, and $A_{r, k}(r=2, \cdots, M), \varphi_{r}$ denote the amplitude and initial phase angle of the $r$ th harmonic component. $v_{k}$ represents a zero-mean white noise with unknown covariance $\mathrm{E}\left(v_{k} v_{k}^{\mathrm{T}}\right)=R_{k} . M$ is the highest order of the harmonic component, and $T_{s}$ is the sampling interval, which can be obtained as $1 / f_{s}$ ( $f_{s}$ is the sampling frequency).

To detect the variation of the amplitude using the EKF, unknown states variables $\mathbf{X}_{k}=\left[x_{1, k}, x_{2, k}, \cdots, x_{2 r-1, k}, x_{2 r, k}\right.$, $\left.\cdots, x_{2 M-1, k}, x_{2 M, k}\right]^{\mathrm{T}}$ are assumed as

$$
\begin{aligned}
& x_{1, k}=A_{1, k} \cos \left(\omega k T_{s}+\varphi_{1}\right), \quad \vdots \\
& x_{2 r-1, k}=A_{r, k} \cos \left(r \omega k T_{s}+\varphi_{r}\right), \quad x_{2 r, k}=A_{r, k} \sin \left(r \omega k T_{s}+\varphi_{r}\right) \\
& x_{2 M-1, k}=A_{M, k} \cos \left(M \omega k T_{s}+\varphi_{M}\right), \\
& x_{2 M, k}=A_{M, k} \sin \left(M \omega k T_{s}+\varphi_{M}\right)
\end{aligned}
$$

By considering state variables above, model (1) can be rewritten in the form of state space as follows 
$$
\left\{\begin{aligned}
\mathbf{X}_{k} & =\mathbf{F X}_{k-1}+\boldsymbol{\eta}_{k} \\
& =\left[\begin{array}{ccccc}
\mathbf{F}_{1} & 0 & \cdots & 0 & 0 \\
0 & \ddots & 0 & \cdots & 0 \\
0 & \cdots & \mathbf{F}_{r} & 0 & 0 \\
0 & \cdots & 0 & \ddots & 0 \\
0 & \cdots & \cdots & 0 & \mathbf{F}_{M}
\end{array}\right]\left[\begin{array}{c}
x_{1, k-1} \\
x_{2, k-1} \\
\vdots \\
x_{2 r-1, k-1} \\
x_{2 r, k-1} \\
\vdots \\
x_{2 M-1, k-1} \\
x_{2 M, k-1}
\end{array}\right]+\boldsymbol{\eta}_{k} \boldsymbol{\eta}_{k} \sim N\left(0, \mathbf{Q}_{k}\right) \\
y_{k}= & \mathbf{H X}_{k}+v_{k}
\end{aligned}\right.
$$

Here, $\mathbf{F}, \mathbf{H}$ denote the system matrix and the observation matrix, respectively. $\boldsymbol{\eta}_{k}$ and $v_{k}$ are the process noise and the observation noise, which are zero-mean Gaussian random variables with covariance $\mathbf{Q}_{k}$ and $R_{k}$, respectively. And the matrices $\mathbf{F}_{r}, \mathbf{H}, \mathbf{Q}_{k}, R_{k}$ are defined as

$$
\begin{aligned}
& \mathbf{F}_{r}=\left[\begin{array}{cc}
\cos \left(r \omega T_{s}\right) & -\sin \left(r \omega T_{s}\right) \\
\sin \left(r \omega T_{s}\right) & \cos \left(r \omega T_{s}\right)
\end{array}\right] \quad(r=1, \cdots M) \\
& \mathbf{H}=\left[\begin{array}{llllllll}
1 & 0 & \cdots & 1 & 0 & \cdots & 1 & 0
\end{array}\right] \\
& \mathbf{Q}_{k}=\operatorname{diag}\left(\left[\gamma_{1}^{2}, \gamma_{2}^{2}, \cdots, \gamma_{2 r-1}^{2}, \gamma_{2 r}^{2}, \cdots, \gamma_{2 M-1}^{2}, \gamma_{2 M}^{2}\right]\right) \\
& R_{k}=\left[\varepsilon^{2}\right]
\end{aligned}
$$

In Eq. (4), all the unknown constant parameters $\left(\gamma_{1}, \gamma_{2}, \cdots, \gamma_{2 M-1}, \gamma_{2 M}, \varepsilon\right)$ should be optimized.

Thus, the amplitude of each frequency component can be calculated as

$$
A_{r, k}=\sqrt{\left(x_{2 r-1, k}\right)^{2}+\left(x_{2 r, k}\right)^{2}}
$$

Where $x_{2 r-1, k}, x_{2 r, k}$ can be obtained as estimated states from model (3).

\section{The EKF-ML Method}

The purpose here is to determine all the unknown constant parameters and to estimate the state $\mathbf{X}_{k}$ from the noisy observations using model (3). This is a type of nonlinear filtering problem, for which we use the EKF to estimate the state from the noisy observations. However, the practical application of the EKF has been limited by the difficulties in selecting the process noise parameter $\mathbf{Q}_{k}$ and the observation noise parameter $R_{k}$, which affects seriously the rate of convergence and the estimation performance. Thus, to obtain the best estimation, it is proposed in this paper to use the maximum likelihood method for the optimal choice of the noise covariance matrices $\mathbf{Q}_{k}$ and $R_{k}$.

\subsection{State estimation by the extended Kalman filter}

Let $\hat{\mathbf{X}}_{k \mid k-1}, \mathbf{S}_{k \mid k-1}$ denote the conditional mean and conditional covariance of $\mathbf{X}_{k}$ given $y_{1: k-1}=\left\{y_{1}, \cdots, y_{k-1}\right\}$, $\hat{v}_{k}$ be the predicted innovation of $y_{k}$. Therefore, the prediction equations using the recursive extended Kalman filter may be given as follows

$$
\left\{\begin{array}{l}
\hat{\mathbf{X}}_{k \mid k-1}=\mathrm{E}\left\{\mathbf{X}_{k} \mid y_{1: k-1}\right\}=\mathbf{F} \hat{\mathbf{X}}_{k-1 \mid k-1} \\
\hat{v}_{k}=y_{k}-\mathbf{H} \hat{\mathbf{X}}_{k \mid k-1} \\
\mathbf{S}_{k \mid k-1}=\mathrm{E}\left\{\left(\mathbf{X}_{k}-\hat{\mathbf{X}}_{k \mid k-1}\right)\left(\mathbf{X}_{k}-\hat{\mathbf{X}}_{k \mid k-1}\right)^{\mathrm{T}}\right\}=\mathbf{F} \mathbf{S}_{k-1 \mid k-1} \mathbf{F}^{\mathrm{T}}+\mathbf{Q}_{k}
\end{array}\right.
$$

Let $\hat{\mathbf{X}}_{k \mid k}, \mathbf{S}_{k \mid k}$ denote the conditional mean and conditional covariance of $\mathbf{X}_{k}$ given $y_{1: k}=\left\{y_{1}, \cdots, y_{k}\right\}$, $\hat{\Psi}_{k}$ be the covariance of $\mathrm{V}_{k}$, then the filtered state is given as

$$
\left\{\begin{array}{l}
\hat{\mathbf{X}}_{k \mid k}=\mathrm{E}\left\{\mathbf{X}_{k} \mid y_{1: k}\right\}=\hat{\mathbf{X}}_{k \mid k-1}+\mathbf{K}_{k} \hat{v}_{k} \\
\mathbf{S}_{k \mid k}=\mathrm{E}\left\{\left(\mathbf{X}_{k}-\hat{\mathbf{X}}_{k \mid k}\right)\left(\mathbf{X}_{k}-\hat{\mathbf{X}}_{k \mid k}\right)^{\mathrm{T}}\right\}=\left[\mathbf{I}-\mathbf{K}_{k} \mathbf{H}\right] \mathbf{S}_{k \mid k-1} \\
\hat{\Psi}_{k}=\mathrm{E}\left\{\hat{v}_{k} \hat{v}_{k}^{\mathrm{T}}\right\}=\mathbf{H} \mathbf{S}_{k \mid k-1} \mathbf{H}^{\mathrm{T}}+\mathbf{R}_{k} \\
\mathbf{K}_{k}=\mathbf{S}_{k \mid k-1} \mathbf{H}^{\mathrm{T}} \hat{\Psi}_{k}^{-1}
\end{array}\right.
$$

\subsection{Parameter estimation by the maximum likelihood}

In Eqs. (6)-(7), except for the constant parameters $\gamma_{1}, \gamma_{2}, \cdots, \gamma_{2 M-1}, \gamma_{2 M}, \varepsilon$, the initial state $\mathbf{X}_{0 \mid 0}$ and the

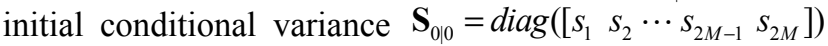
also need to be estimated. So the parameters to be estimated are $\theta=\left(\gamma_{1}, \cdots, \gamma_{2 M}, \mathcal{\varepsilon}, x_{1,0}, \cdots, x_{2 M, 0}, s_{1}, \cdots, s_{2 M}\right)$. Assuming $v_{k}$ is a Gaussian white noise vector with covariance $\Psi_{k}$, then the joint conditional density of $v_{k}$ may be written as

$$
p\left(v_{k} \mid y_{k-1}, \ldots, y_{1}, \theta\right)=\frac{1}{2 \pi\left|\Psi_{k}\right|^{1 / 2}} \exp \left(-\frac{1}{2} v_{k}^{\mathrm{T}} \Psi_{k}^{-1} v_{k}\right)
$$

where $|\bullet|$ denotes the determinant.

In the second equation of model (3), the innovation $v_{k}$ and the observation $y_{k}$ are Gaussian random variables with the same variance but different means. Therefore, $(-2)$ log-likelihood of model (3) may be derived from Eq. (8) as follow

$$
\begin{aligned}
& (-2) \log p\left(y_{N}, \cdots, y_{1} \mid \theta\right) \\
& =\sum_{k=1}^{N}(-2) \log p\left(y_{k} \mid y_{k-1}, \cdots, y_{1}, \theta\right) \\
& =\sum_{k=1}^{N}(-2) \log p\left(v_{k} \mid y_{k-1}, \cdots, y_{1}, \theta\right) \\
& =\sum_{k=1}^{N}\left\{\log \left|\Psi_{k}\right|+v_{k}^{\mathrm{T}} \Psi_{k}^{-1} v_{k}\right\}+N \log 2 \pi
\end{aligned}
$$


where $N$ represents sampling length.

From the estimated innovation $\hat{v}_{k}$ and its covariance $\hat{\Psi}_{k}$ with respect to the given parameters $\theta$, the optimal parameters $\theta^{*}$ may be obtained by minimizing the $(-2)$ $\log$-likelihood function (9) as follow

$$
\begin{array}{r}
\theta^{*}=\arg \min _{\theta} \sum_{k=1}^{N}\left\{\log \left|\hat{\Psi}_{k}(\theta)\right|+\left(\hat{v}_{k}(\theta)\right)^{\mathrm{T}}\left(\hat{\Psi}_{k}(\theta)\right)^{-1}\left(\hat{v}_{k}(\theta)\right)\right\} \\
+N \log 2 \pi
\end{array}
$$

In this paper, the function "FMINSEARCH" based on the Nelder-Mead method in the MATLAB Optimization Toolbox is used to carry out the parameter optimization

\section{Simulations}

To verify the superiority of the proposed algorithm, the conventional EKF, the WT and the proposed EKF-ML are used to detect voltage sag with three kind of simulated signals. The first signal is a pure sinusoidal signal distorted with voltage sag in presence of additive white Gaussian noise. On the basis of the first signal, the second and the third add a transient pulse and higher order harmonics, respectively.

\section{Case 1. A pure sinusoidal signal contaminated noises}

The source voltage in this case takes the following waveform equation:

$$
y_{t}=E_{t} \cos (2 \pi f t+\varphi)+v_{t}
$$

Here, the fundamental frequency $f$ and the voltage amplitude $E_{t}$ are assumed $50 \mathrm{~Hz}(f=50 \mathrm{~Hz})$ and $1 \mathrm{pu}$ ( $E_{t}=1 \mathrm{p} . \mathrm{u}$ ), respectively. Also, constant phase $\varphi$ and sampling time $T_{s}$ are $0 \mathrm{rad}(\varphi=0 \mathrm{rad})$ and $100 \mu \mathrm{s}$ $\left(T_{s}=100 \mu \mathrm{s}\right)$ respectively. $v_{t}$ represents a Gaussian white noise with signal-to-noise ratio (SNR) values of $40 \mathrm{~dB}$, $30 \mathrm{~dB}$ and $20 \mathrm{~dB}$, which means the Gaussian white noise has 0.01p.u, 0.0316p.u and 0.1p.u standard deviation, respectively. In this simulation, when the voltage sag occurs, fundamental amplitude drops to $0.5 \mathrm{p} . \mathrm{u}$, and the time of variation amplitude is considered as below:

$$
E_{t}=\left\{\begin{array}{cc}
1.0 & 0 \mathrm{~s} \leq t<0.05 \mathrm{~s} \\
0.5 & 0.05 \mathrm{~s} \leq t \leq 0.12 \mathrm{~s} \\
1.0 & 0.12 \mathrm{~s}<t \leq 0.2 \mathrm{~s}
\end{array}\right.
$$

Obviously, the time when the voltage sag occurs is from 0.05 s to $0.12 \mathrm{~s}$ in Eq. (12).

Fig. 1 shows the observed sinusoidal signals with changes in noise and the clean sinusoidal signal without noise. Figs. 2-4 show comparisons of the actual vs. estimated results in terms of the amplitude and the signal using the proposed EKF-ML and the conventional EKF algorithms at $40 \mathrm{~dB}, 30 \mathrm{~dB}$ and $20 \mathrm{~dB}$ SNR's noise, respectively. Table 1 gives comparisons of the actual parameter $\mathcal{E}$ vs. the estimated parameter by the EKF-ML. For testing the performance of WT, daubechies discrete wavelet of order $4(\mathrm{db} 4)$ is used for PQ disturbance signal analysis because daubechies wavelets are the family of orthogonal wavelets and the $\mathrm{db} 4$ exhibits more efficient in

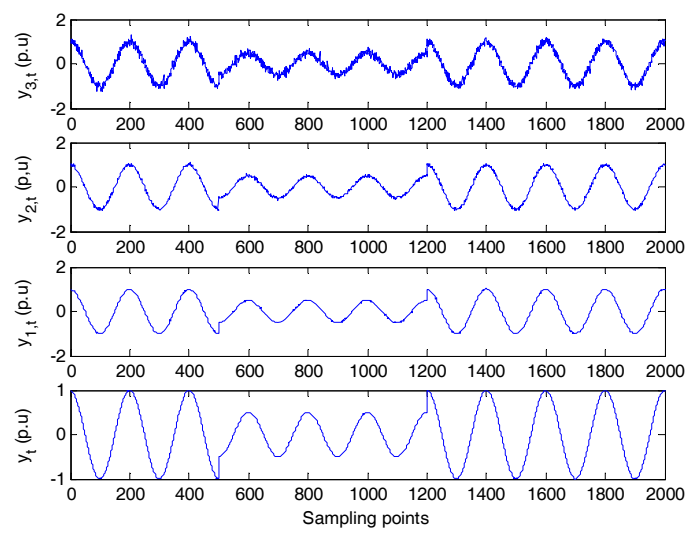

Fig. 1. The observed sinusoidal signals and the clean sinusoidal signal $\left(y_{t}, y_{1, t}, y_{2, t}, y_{3, t}\right.$ denote the clean signal without noise and the observed signals disturbed with SNR values of $40 \mathrm{~dB}, 30 \mathrm{~dB}$ and 20 $\mathrm{dB}$, respectively).
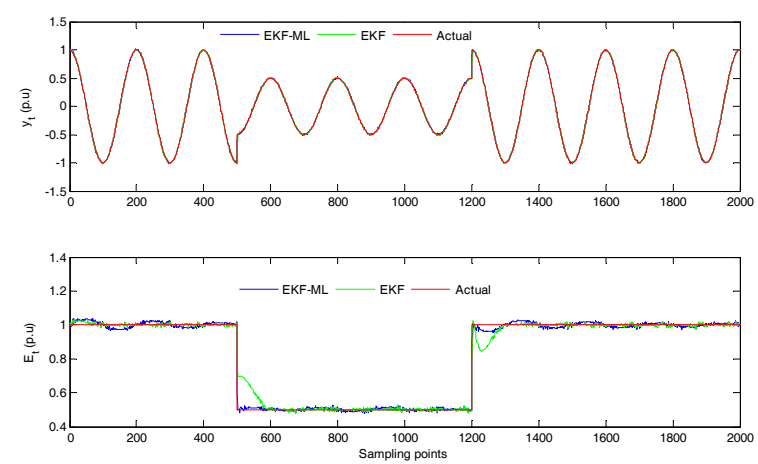

Fig. 2. The estimated signal and amplitude using the EKFML and the EKF at $40 \mathrm{~dB}$ SNR's noise.
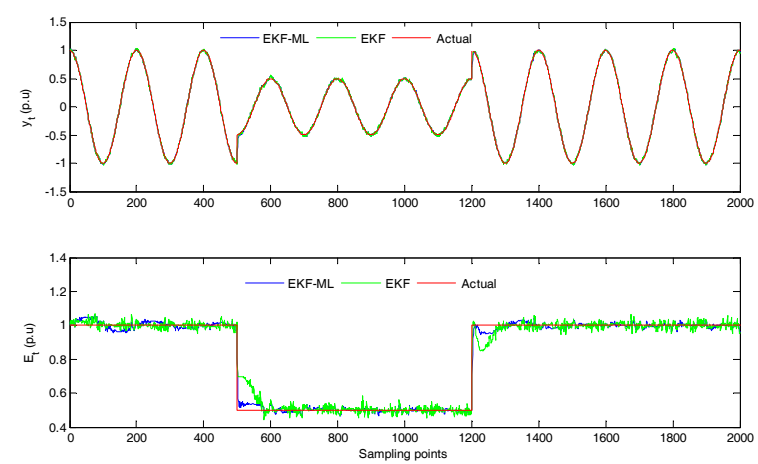

Fig. 3. The estimated signal and amplitude using the EKFML and the EKF at $30 \mathrm{~dB}$ SNR's noise 
feature extraction. Firstly, the observed signal is denoised by wavelet threshold to remove random noise from the original signal. After denoising, signal can be reconstructed using inverse discrete wavelet transform. Then, 4 layer daubechies wavelet decomposition is applied to the signal that has been denoised. Fig. 5 gives the signal comparisons between the actual and the reconstructed after denoising by WT. Figs. 6-8 show detail coefficients of four layer WT decomposition process.

At the top of Figs. 2-4, it is clear that the estimated signals by the EKF-ML closely match with the actual signals in all conditions. Also, the estimated amplitudes changing in time by the EKF-ML are very close to the actual amplitudes in all situations, although the former have slight fluctuations around the latter. On the other hand, under the conditions of $40 \mathrm{~dB}$ and $30 \mathrm{~dB}$ SNR, the estimated signals by the EKF match with the actual signals

Table 1. The estimated parameter $\varepsilon$ with the EKF-ML

\begin{tabular}{ccccc}
\hline Noise & Case 1 & Case 2 & Case 3 & Actual \\
\hline $40 \mathrm{~dB}$ & 0.00958 & 0.00970 & 0.0107 & 0.0100 \\
$30 \mathrm{~dB}$ & 0.0321 & 0.0297 & 0.0313 & 0.0316 \\
$20 \mathrm{~dB}$ & 0.0986 & 0.101 & 0.100 & 0.100 \\
\hline
\end{tabular}

Note: Case 1, Case 2, and Case 2 represent three kinds of signals generated by model (11), model (13) and model (14) respective ly in Tables 1-3. Furthermore, each kind of signal (Case 1, Ca se 2 , or Case 2) is disturbed by the white noise with three dif ferent levels, namely, $40 \mathrm{~dB}, 30 \mathrm{~dB}, 20 \mathrm{~dB}$.
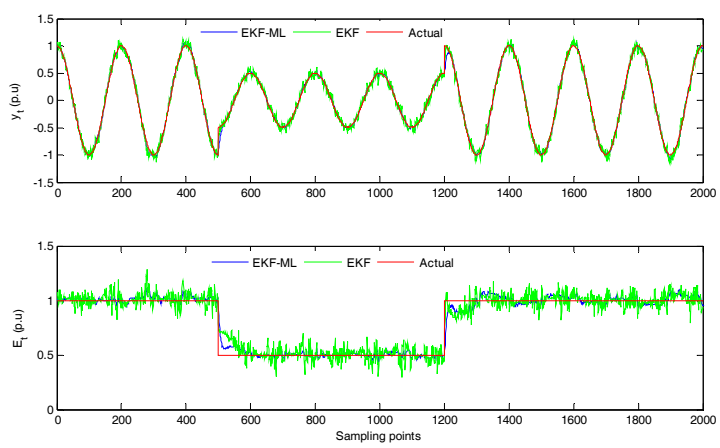

Fig. 4. The estimated signal and amplitude using the EKFML and the EKF at $20 \mathrm{~dB}$ SNR's noise
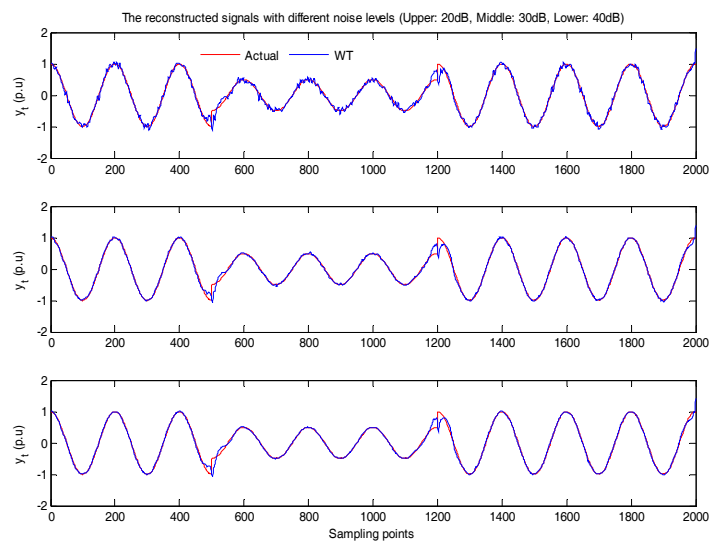

Fig. 5. The denoised and reconstructed signals by the WT as closely as the EKF-ML. However, when SNR value drops to $20 \mathrm{~dB}$, the estimated signals by the EKF deviate appreciably from the actual signals. In terms of the estimated amplitudes, the EKF has relatively worse accuracy than the EKF-ML, especially when the voltage sag occurs. As shown in Figs. 2-4, in every case, the EKF-ML algorithm can detect the voltage sag in real time and has fast response to the voltage sag. Instead, the EKF exhibits a long response time. By comparing Figs. 2-4, as SNR value decreases from $40 \mathrm{~dB}$ to $20 \mathrm{~dB}$, there are more deviations of the estimated value from the actual values, and the estimated amplitude fluctuations slowly become great for the two methods. This result shows that the noise strength slightly affects the estimated performance of the two methods in the case of a pure sinusoidal signal. In Table 1, it can be seen that the values of the estimated parameter $\varepsilon$ by the EKF-ML are very close to the actual values in all conditions, while the conventional EKF needs a given noise parameter value. Thus, the accurate estimation of the observation noise covariance matrix can further ensure that the EKF has better accuracy and faster convergence. This also verifies the effectiveness and the accuracy of the proposed algorithm.

In Fig. 5, we can see the SNR of the reconstructed signal

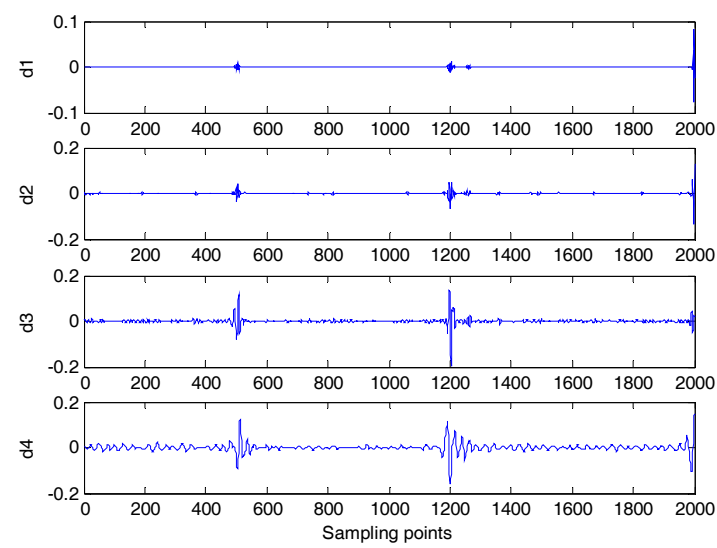

Fig. 6. Four layer daubechies wavelet decomposition at 40 dB SNR

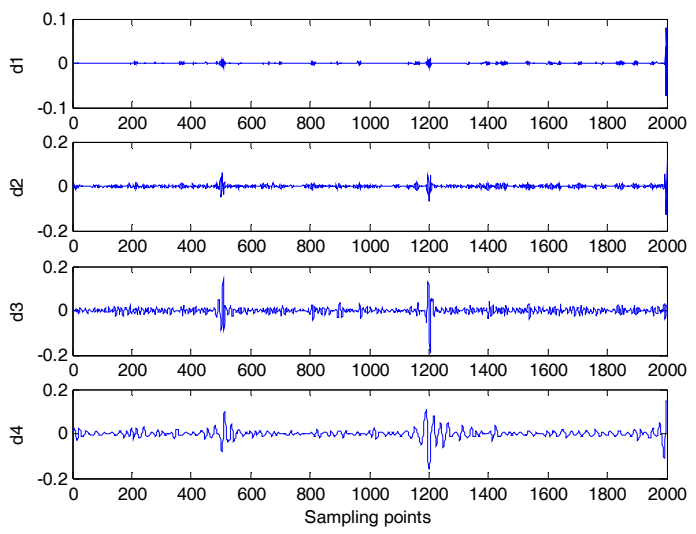

Fig. 7. Four layer daubechies wavelet decomposition at 30 dB SNR 
by WT has been improved and denoising effect is relatively obvious. However, the reconstructed signals by the WT deviate appreciably from the actual signals, especially in the vicinity of sample points 500 and 1200, in which the voltage sag occurs. From this point, the estimated signals by the EKF-ML shown in Figs. 2-4 match the clean (actual) signals better than the reconstructed signal by WT shown in Fig. 5. As see from Figs. 6-7, the wavelet coefficients $\mathrm{d} 3, \mathrm{~d} 4$ reach peaks at the start and end of the voltage sag, while the wavelet coefficients in Fig. 8 appear obvious oscillations and do not give significant peaks at the start and end of the voltage sag. This underlines that the performance of $\mathrm{db} 4$ to detect voltage sag becomes worse with signal noise ratio decreasing. Thus, compared with the EKF-ML, the WT can't give precise and quick response to voltage sag when the signals are heavily contaminated by random noise.

To compare the performance of the proposed method with the conventional EKF approach and the WT method, the RMSE (root mean square error) values of the estimated waveform (or the reconstructed signals) with respect to the actual waveform without noise are computed and compared in Table 2. Also, Table 3 lists the running time for the EKF-ML and EKF methods. It is clear that the RMSE values of the EKF-ML in every case are lower than those of the EKF and much lower than those of the WT. Moreover, with decreasing SNR, the RMSE value of the EKF-ML is lower and lower than that of the EKF. Instead,

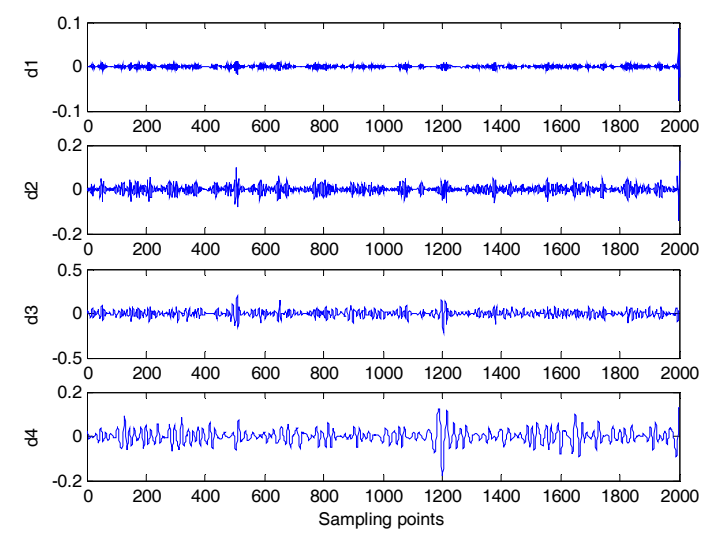

Fig. 8. Four layer daubechies wavelet decomposition at 20 dB SNR

Table 2. The RMSE for the EKF-ML and the EKF under three conditions

\begin{tabular}{ccccc}
\hline Case & Method & $\begin{array}{c}\text { Noise 1 } \\
40 \mathrm{~dB}\end{array}$ & $\begin{array}{c}\text { Noise 2 } \\
\text { 30dB }\end{array}$ & $\begin{array}{c}\text { Noise 3 } \\
\text { 20dB }\end{array}$ \\
\hline \multirow{3}{*}{ Case 1 } & EKF-ML & 0.0182 & 0.0248 & 0.0432 \\
& EKF & 0.0193 & 0.0260 & 0.0641 \\
& WT & 0.0648 & 0.0656 & 0.0811 \\
Case 2 & EKF-ML & 0.0109 & 0.0174 & 0.0258 \\
& EKF & 0.0110 & 0.0216 & 0.0381 \\
& WT & 0.0382 & 0.0415 & 0.0625 \\
Case 3 & EKF-ML & 0.0246 & 0.0335 & 0.0534 \\
& EKF & 0.0252 & 0.0403 & 0.0838 \\
& WT & 0.2477 & 0.2479 & 0.2583 \\
\hline
\end{tabular}

the RMSE value of the WT changes only a little with decreasing SNR. All this indicates that the EKF-ML can obtain better estimation performance than the conventional EKF and the WT. Moreover, this demonstrates the WT method is not enough fine in time domain and has bad time resolution. In Table 3, we can conclude that the EKF-ML and EKF methods have relatively short running time even though the running time of the EKF shortens greatly.

\section{Case 2. Signal contaminated with a pulse and a gaussian noise}

In this case, the signal used for the estimation, besides the fundamental frequency, contains a pulse and is simultaneously disturbed with a Gaussian white noise. This kind of signal is considered as follow

$$
y_{t}=\left\{\begin{array}{l}
\cos (2 \pi f t+\varphi)+v_{t} 0 \mathrm{~s} \leq t<0.0803 \mathrm{~s} \text { and } 0.0823 \mathrm{~s}<t \leq 0.15 \mathrm{~s} \\
\cos (2 \pi f t+\varphi)+0.5 e^{-10 t}+v_{t} \quad 0.0803 \mathrm{~s} \leq t \leq 0.0823 \mathrm{~s}
\end{array}\right.
$$

Here, the fundamental frequency, the constant phases $\varphi$, the sampling time $T_{s}$ and the noise $v_{t}$ are the same as Case 1.

Fig. 9 shows the observed signals, which are contaminated with a pulse and a Gaussian noise, and the clean signal without noise but with a pulse. In Fig. 9, we can clearly see a sudden and small change in the waveform. Figs. 10-12 show comparisons of the estimated signals and amplitudes vs. the actual signals and amplitudes under different conditions. Fig. 13 gives the signal comparisons between the actual and the reconstructed after denoising by WT. Figs. 14-16 show detail coefficients of four layer WT decomposition process. The estimated parameter $\mathcal{E}$ by the EKF-ML is given in Table 1.

In the upper part of Figs.10-12, the estimated signals by the EKF-ML can track the actual signals accurately except

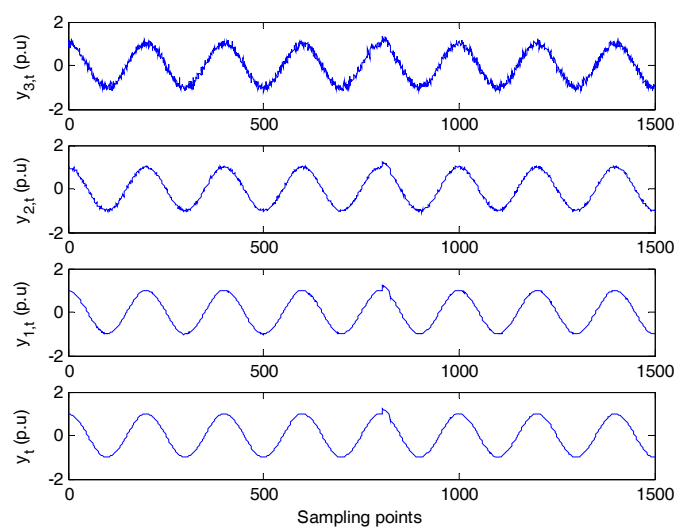

Fig. 9. The observed signals and the clean signal $\left(y_{t}, y_{1, t}, y_{2, t}, y_{3, t}\right.$ denote the clean signal and the observed signals disturbed with SNR values of 40 $\mathrm{dB}, 30 \mathrm{~dB}$ and $20 \mathrm{~dB}$, respectively) 

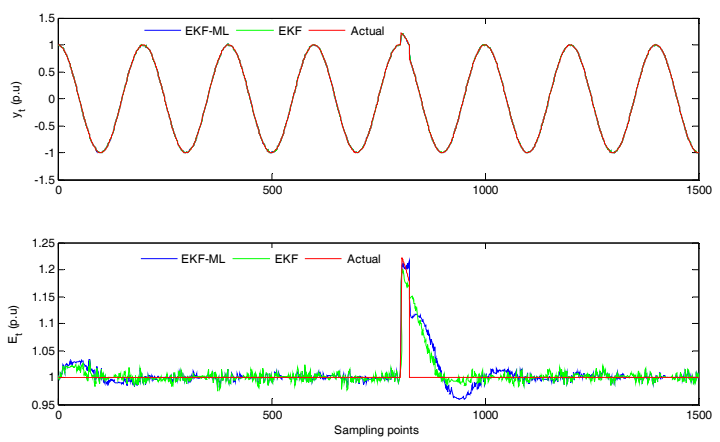

Fig. 10. The estimated signal and amplitude using the adaptive EKF at $40 \mathrm{~dB}$ SNR's noise
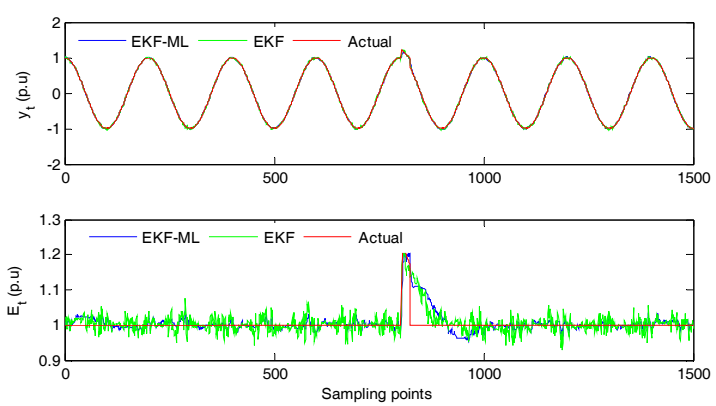

Fig. 11. The estimated signal and amplitude using the adaptive EKF at $30 \mathrm{~dB}$ SNR's noise
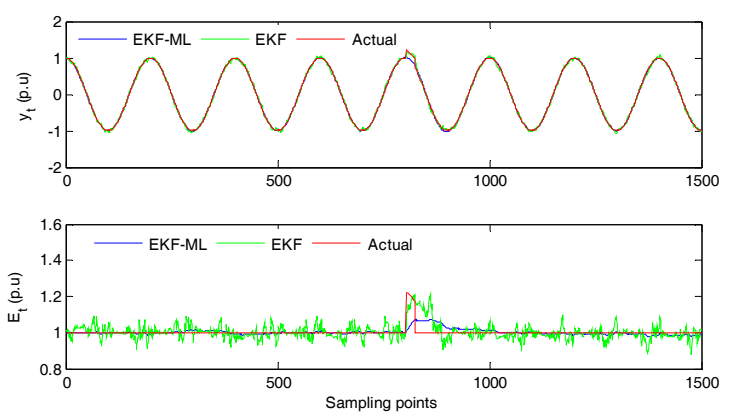

Fig. 12. The estimated signal and amplitude using the adaptive EKF at $20 \mathrm{~dB}$ SNR's noise

that there are small deviations of the estimated value from the actual value in the case of $20 \mathrm{~dB}$ SNR. In the lower part of Figs. 10-12, the proposed EKF-ML algorithm can accurately capture the pulse, and the estimated amplitudes roughly match the actual amplitudes in all conditions. Obviously, when a pulse occurs in these conditions of 40 $\mathrm{dB}$ and $30 \mathrm{~dB}$ SNR, the EKF-ML algorithm can fast and accurately track the rising edge, but it exhibits relatively long response time in tracking the falling edge. Although the estimated peak is lower than the actual peak in the condition of $20 \mathrm{~dB}$ SNR, the response speed is as fast as the former two. As for the EKF, the estimated signals match with the actual signals as closely as the EKF-ML, but the accuracy of estimated amplitudes is lower than that of the EKF-ML, especially when a pulse occurs. In Fig. 13, we can see the SNR of the reconstructed signal by WT has
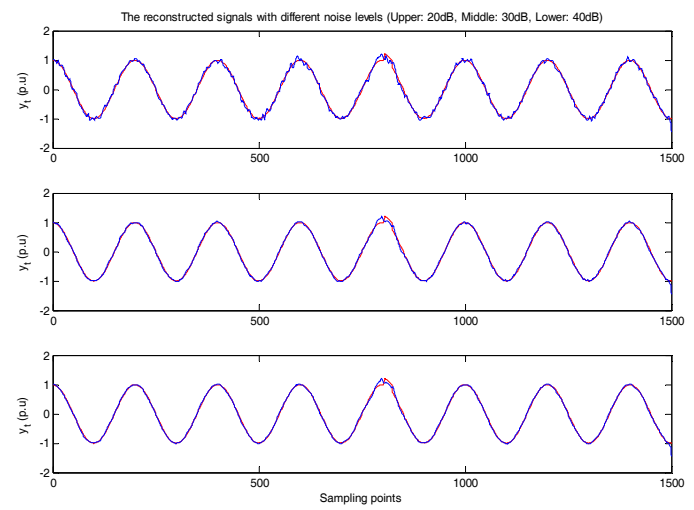

Fig. 13. The denoised and reconstructed signals by the WT

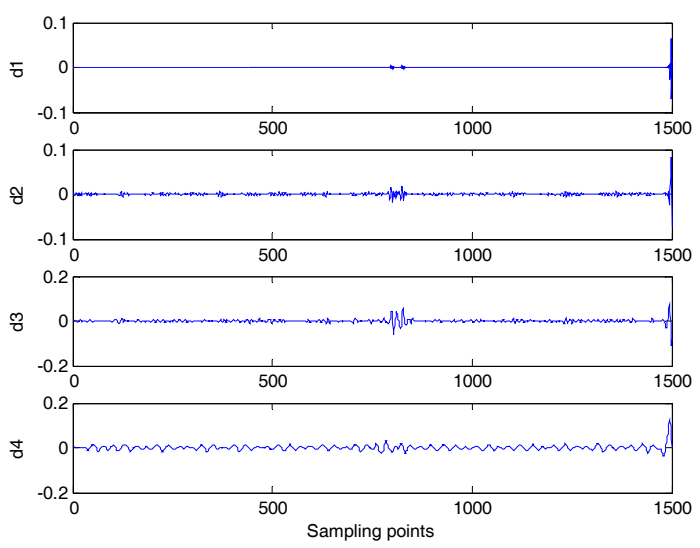

Fig. 14. Four layer daubechies wavelet decomposition at $40 \mathrm{~dB}$ SNR

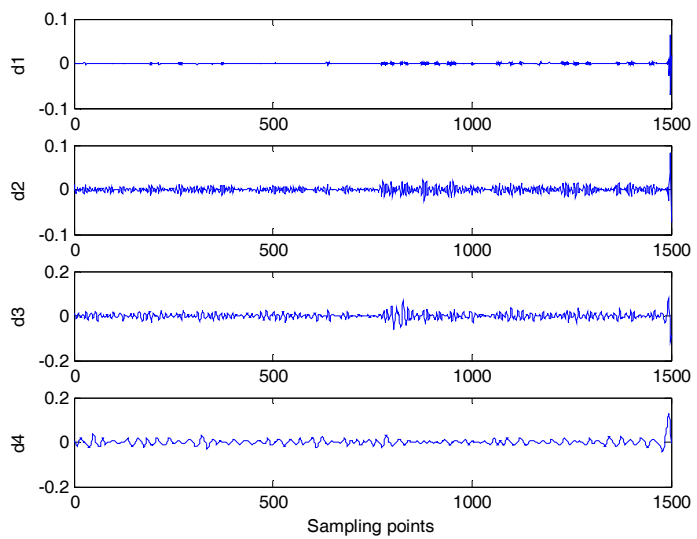

Fig. 15. Four layer daubechies wavelet decomposition at $30 \mathrm{~dB}$ SNR

been greatly improved and denoising effect is obvious. The reconstructed signals by the WT match the actual signals well, except when a pulse occurs. From this point, the reconstructed signal by WT shown in Fig. 13 match the clean (actual) signals as well as the estimated signals by the EKF-ML shown in Figs 10-12. As see from Fig. 14, the wavelet coefficients $\mathrm{d} 2, \mathrm{~d} 3$ reach small peaks in the vicinity of a pulse, while the wavelet coefficients in Figs. 15-16 appear obvious oscillations and do not give 
significant peaks at the start and end of the voltage sag (a pulse). This underlines that the WT (db4) can't perform accurately for detection of sudden or fast changes in waveform e.g. transient or a pulse. Also, the performance of $\mathrm{db} 4$ to detect voltage sag becomes rather worse with signal noise ratio decreasing and deteriorates greatly compared with the EKF-ML. In Table 1, we can see that the values of the estimated parameter $\varepsilon$ are very close to the actual values in all conditions, which is the same as Case 1.The RMSE values shown in Table 2 also indicate the EKF-ML is superior to the EKF and the WT.

\section{Case 3. Signal contaminated with harmonics and noises}

In this case, the signal used for the estimation, besides the fundamental frequency, contains higher harmonics of the $3 \mathrm{rd}$, 5rd and is simultaneously contaminated with a Gaussian white noise. This kind of signal is represented as follow

$$
\begin{aligned}
y_{t}= & E_{1, t} \cos \left(2 \pi f t+\varphi_{1}\right)+E_{3, t} \cos \left(6 \pi f t+\varphi_{3}\right) \\
& +E_{5, t} \cos \left(10 \pi f t+\varphi_{5}\right)+v_{t}
\end{aligned}
$$

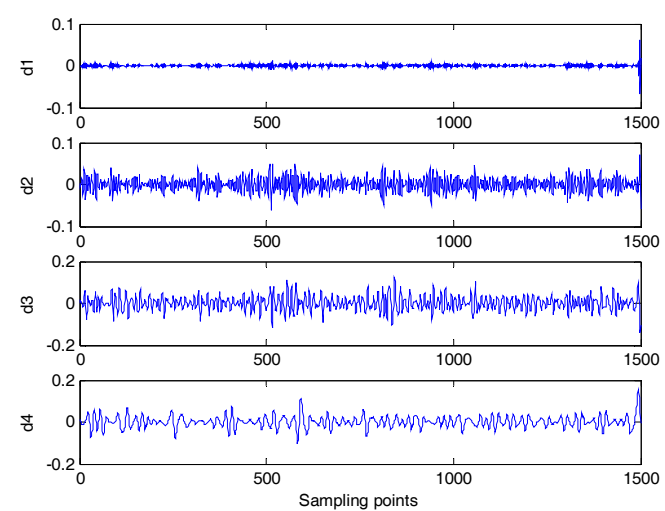

Fig. 16. Four layer daubechies wavelet decomposition at $20 \mathrm{~dB}$ SNR
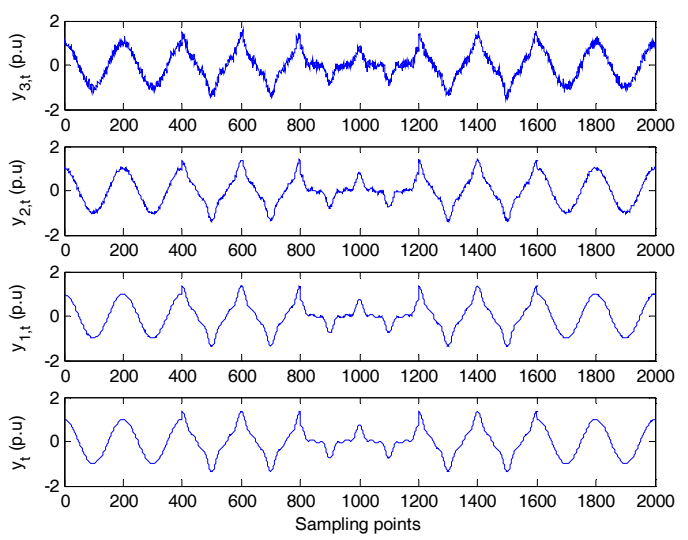

Fig. 17. The observed signals and the clean signal $\left(y_{t}, y_{1, t}, y_{2, t}, y_{3, t}\right.$ denote the clean signal and the observed signals disturbed with SNR values of 40 $\mathrm{dB}, 30 \mathrm{~dB}$ and $20 \mathrm{~dB}$, respectively)
Here, the fundamental frequency, the sampling time $T_{s}$ and the noise $v_{t}$ are the same as Case 1, and the constant phases $\varphi_{i}(i=1,3,5)$ are assumed 0rad. The voltage amplitudes $E_{i, t}(i=1,3,5)$ are considered as follows

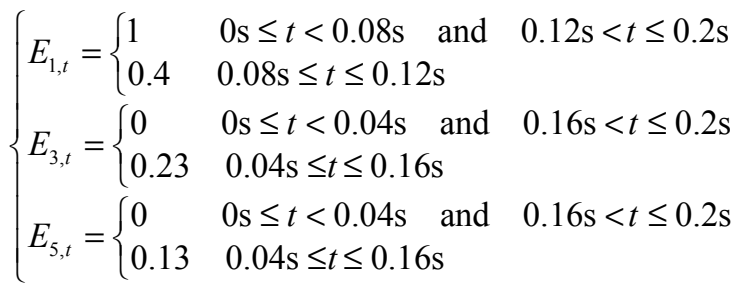

In Eq. (15), the time when the voltage sag of the fundamental frequency occurs is from $0.08 \mathrm{~s}$ to $0.12 \mathrm{~s}$, and the time when the perturbations of the harmonics occur is from $0.04 \mathrm{~s}$ to $0.16 \mathrm{~s}$.

Fig. 17 shows the observed signals, which are contaminated with harmonics and noises, and the clean signal without noise. Figs. 18-20 show comparisons of the estimated signals and amplitudes vs. the actual signal and amplitude for the two methods as the SNR decreases from $40 \mathrm{~dB}$ to $20 \mathrm{~dB}$. Fig. 21 gives the signal comparisons between the actual and the reconstructed after denoising by

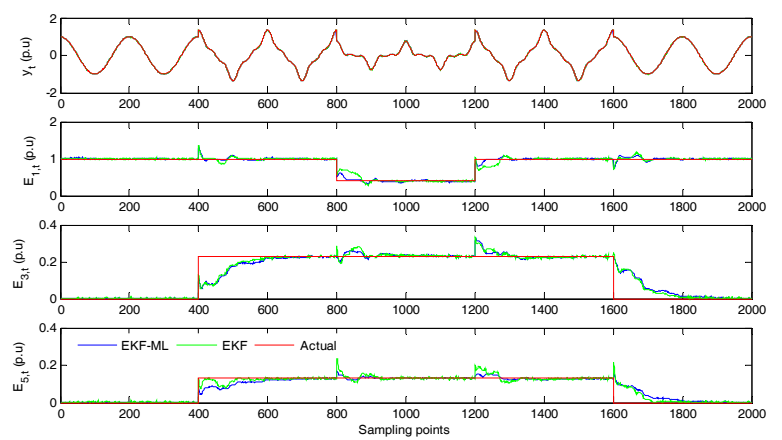

Fig. 18. The estimated signals and amplitudes using the adaptive EKF at $40 \mathrm{~dB}$ SNR's noise $\left(E_{1, t}, E_{3, t}\right.$, $E_{5, t}$ denote the amplitudes of the fundamental frequency, the $3 \mathrm{rd}$ harmonic and the 5th harmonic, respectively)
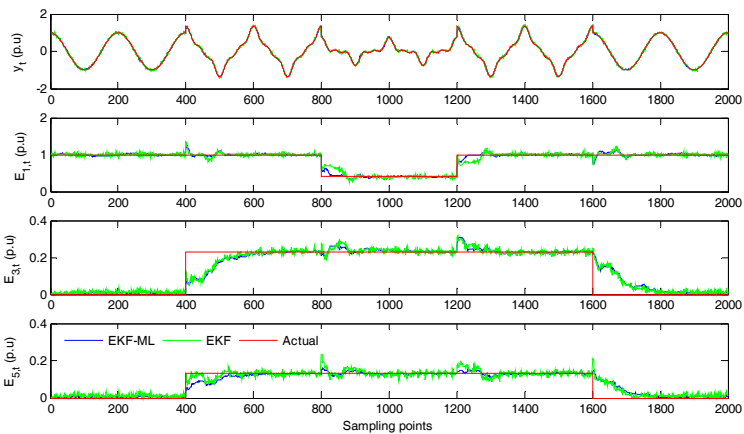

Fig. 19. The estimated signals and amplitudes using the adaptive EKF at $30 \mathrm{~dB}$ SNR's noise 
WT. Figs. 22-24 show detail coefficients of four layer WT decomposition process. The estimated parameter $\mathcal{E}$ by the EKF-ML is given in Table 1.

In Figs. 18-20, as for the EKF-ML, the estimated signals closely match the actual signal in all conditions even though the observed signals contain the 3rd, 5th harmonics and are simultaneously contaminated with various noise. Also, the estimated amplitudes of the fundamental frequency are all very close to the actual amplitude of the fundamental frequency, and the response to the voltage sage is very fast and accurate in terms of the fundamental frequency.

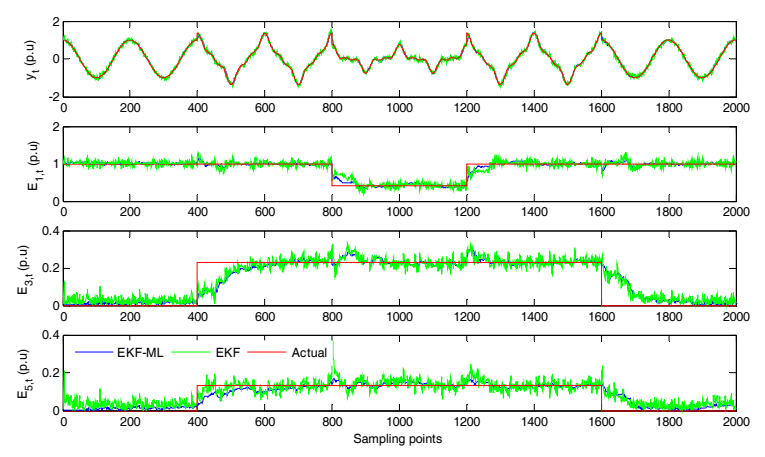

Fig. 20. The estimated signals and amplitudes using the adaptive EKF at $20 \mathrm{~dB}$ SNR's noise
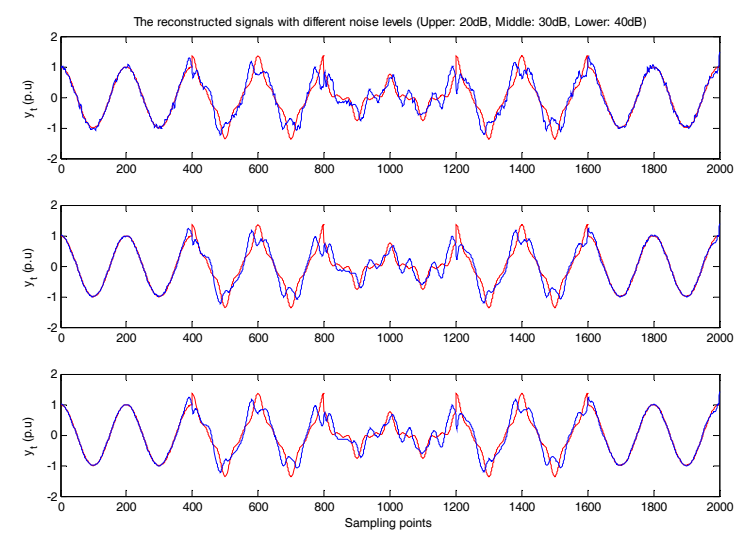

Fig. 21. The denoised and reconstructed signals by the WT

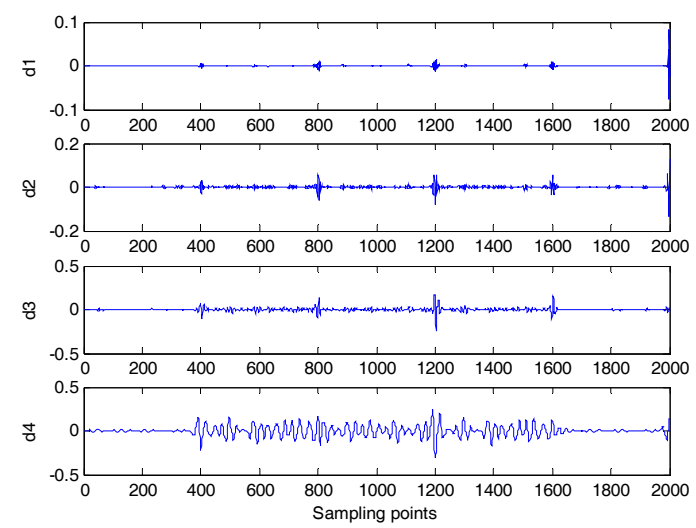

Fig. 22. Four layer daubechies wavelet decomposition at $40 \mathrm{~dB}$ SNR
The EKF-ML algorithm also can successfully detect the harmonics and accurately estimate the amplitudes of the harmonics, although it exhibits some delay in tracking the falling edge relative to the fundamental frequency. By comparing Figs. 18-20, as SNR value decreases from $40 \mathrm{~dB}$ to $20 \mathrm{~dB}$, the estimated amplitude fluctuations gradually become great. This increase in amplitude fluctuation is approximately consistent with that of Case 1, but only it fluctuates acutely relative to Case 1 .

However, as shown in Fig. 21, the reconstructed signals by the WT deviate appreciably from the actual signals because of harmonics interference. In Figs. 22-23, the wavelet coefficients $\mathrm{d} 2, \mathrm{~d} 3$ just reach small peaks at the start and end of the voltage sag and harmonics interference, while the wavelet coefficients in Fig. 24 appear obvious oscillations and do not give significant peaks at the start and end of the voltage sag when the SNR drops to $20 \mathrm{~dB}$. This shows the performance of $\mathrm{db} 4$ to detect voltage sag

Table 3. The running time of different algorithms (Unit: second)

\begin{tabular}{cccc}
\hline Method & Case 1 & Case 2 & Case 3 \\
\hline EKF & 0.0754 & 0.0754 & 0.0914 \\
EKF-ML & 4.96 & 4.87 & 22.4 \\
\hline
\end{tabular}

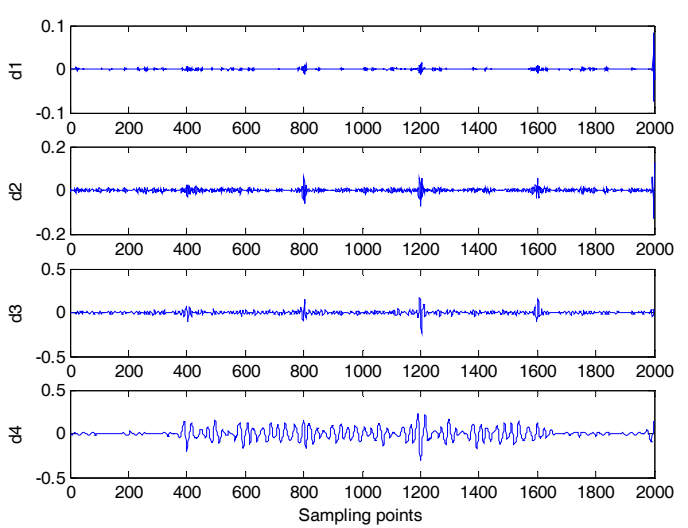

Fig. 23. Four layer daubechies wavelet decomposition at $30 \mathrm{~dB}$ SNR

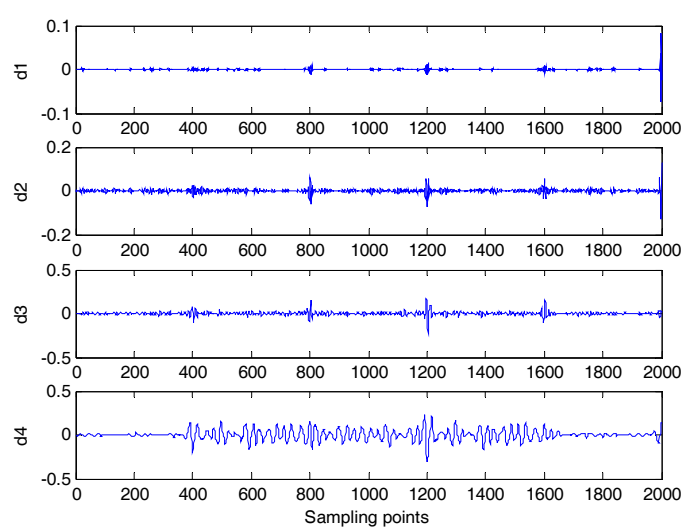

Fig. 24. Four layer daubechies wavelet decomposition at $20 \mathrm{~dB}$ SNR 
becomes worse with signal noise ratio decreasing. Also, compared with the EKF-ML, the WT can't give precise and quick response to voltage sag when the signals are heavily contaminated by random noise and harmonics.

As for the EKF, the estimated signals fit the actual signal as well as the EKF-ML except in cases of $20 \mathrm{~dB}$ SNR, but the estimated amplitudes of the fundamental frequency are less accurate than those of the EKF-ML. In terms of the estimated harmonic amplitudes, the two methods demonstrate almost the same performance except that the EKF exhibits more fluctuations. The RMSE values shown in Table 2 verify the EKF-ML has better estimation performance than the EKF. In this case, the running time of the EKF-ML shown in Table 3 increases rapidly relative to Case 1 and Case 2. This is mainly because the abundant harmonics increase the computation complex greatly.

\section{Conclusion}

In this paper, the adaptive EKF algorithm based on the ML method is proposed to detect the voltage sag. To enhance the estimation performance, the method incorporates the extended Kalman filter and the maximum likelihood method to estimate the states (the voltage amplitude) and noise parameters simultaneously. The complexity of the proposed adaptive algorithm is almost the same as the EKF, which is simpler and less complex than other adaptive algorithms, such as the unscented Kalman smoother algorithm and the adaptive particle swarm optimization (GA-APSO) aided unscented Kalman filter. The EKF-ML algorithm is validated through simulations in these conditions of various noise levels. Simulation results indicate the proposed adaptive algorithm can detect the voltage sag fast and accurately, and can precisely indentify the clean signal, even though the duration of transient voltage pulse is very short or the voltage signal is highly distorted by harmonics and Gaussian noise with various SNR. More importantly, the EKF-ML algorithm is capable of accurately estimating the noise parameters and is robust against various noise levels. The RMSE values indicate that the proposed EKF-ML algorithm is superior to the conventional EKF and the WT, especially in cases of low SNR. Thus, the proposed method is especially appropriate for detection of the voltage sag under the condition of unknown measurement noise.

\section{Acknowledgements}

This work was supported by the National Science Foundation of China $(51507015,61540037,71271215$, 61233008, 51425701, 70921001, 51577014), and the Natural Science Foundation of Hunan Province (2015 JJ3008), and the National key research and development program of China (2016YF0900605).

\section{References}

[1] Ö. Gencer, S. Öztürk, T. Erfidan, "A new approach to voltage sag detection based on wavelet transform," International Journal of Electrical Power \& Energy Systems, vol. 32, no. 2, pp. 133-140, Feb. 2010.

[2] F. B. Costa, B. A. Souza, N. S. D. Brito, "Real-time detection of voltage sags based on wavelet transform," in Proc. IEEE/PES Transmission and Distribution Conference and Exposition, pp.537-542, 2010.

[3] H. M. Beides, G. T. Heydt, "Dynamic state estimation of power system harmonics using Kalman filtering methodology," IEEE Trans. Power Del., vol. 6, no. 4, pp. 1663-1670, Oct. 1991.

[4] R. Naidoo, P. Pillay, "A new method of voltage sag and swell detection," IEEE Trans. Power Del., vol. 22, no. 2, pp. 1056-1063, Apr. 2007.

[5] M. B. Latran, A. Teke, "A novel wavelet transform based voltage sag/swell detection algorithm," International Journal of Electrical Power \& Energy Systems, vol. 71, pp. 131-139, Oct.2015.

[6] S. Suja, J. Jerome, "Pattern recognition of power signal disturbances using $\mathrm{S}$ transform and TT transform," International Journal of Electrical Power \& Energy Systems, vol. 32, no. 1, pp. 37-53, Jan. 2010.

[7] D. M. A. Hussain, R. M. Imran, G. M. Shoro, "Harmonic detection at initialization with Kalman filter," in Proc. The 6th International Congress on Ultra Modern Telecommunications and Control Systems and Workshops, pp. 614-617, Oct. 2014.

[8] P. Nayak, J. M. Patnaik, "Harmonics reckoning in micro grid using Kalman and weighted Kalman filter algorithm," in Proc. International Conference on Electrical, Electronics, Signals, Communication and Optimization (EESCO), pp. 1-4, 2015.

[9] H. K. Sahoo, P. K. Dash, "Robust estimation of power quality disturbances using unscented $\mathrm{H}_{\infty}$ filter," International Journal of Electrical Power \& Energy Systems, col. 73, pp. 438-447, Dec. 2015.

[10] K. K. C Yu, N. R. Watson, J. Arrillaga. "An adaptive Kalman filter for dynamic harmonic state estimation and harmonic injection tracking," IEEE Trans. Power Del., vol. 20, no. 2, pp. 1577-1584, Apr. 2005.

[11] K. R. Shih, S. J. Huang, "Application of a robust algorithm for dynamic state estimation of a power system," IEEE Transactions on Power Systems, vol. 17, no. 1, pp. 141-147, Mar. 2002.

[12] K. Kennedy, G. Lightbody, R. Yacamini, "Power system harmonic analysis using the Kalman filter," in Proc. Power Engineering Society General Meeting, vol. 2, pp. 752-757, 2003.

[13] J. H. Zhang, G. Welch, G. Bishop, Z. Y. Huang, "A two-stage Kalman filter approach for robust and realtime power system state estimation," IEEE Transactions on Sustainable Energy, vol. 5, no. 2, pp. 629636, Apr. 2014. 
[14] R. K. Jatoth, G. A. Reddy, "A hybrid GA-adaptive particle swarm optimization based tuning of unscented Kalman filter for harmonic estimation," in Proc. Swarm, Evolutionary, and Memetic Computing, pp. 380-388, 2010.

[15] R. A. Fisher, "Statistical methods for research workers," Genesis Publishing Private Limited, 1925.

[16] H. Peng, G. Kitagawa, Y. Tamura, et al., "A modeling approach to financial time series based on market microstructure model with jumps," Applied Soft Computing, vol. 29, pp. 40-51, Apr. 2015.

[17] S. Edla, J. J. Zhang, J. Spanias, et al., "Adaptive parameter estimation of cardiovascular signals using sequential Bayesian techniques," in Proc. The Forty Fourth Asilomar Conference on Signals, Systems and Computers (ASILOMAR), pp. 374-378, 2010.

[18] S. Hasan, P. K. Dash, B. K. Panigrahi, et al., “Adaptive unscented filtering technique and particle swarm optimization for estimation of non-stationary signal parameters," in Proc. IEEE Conference on Industrial Electronics and Applications, pp. 3853-3858, 2009.

[19] E. M. Siavashi, A. Rouhani, R. Moslemi. "Detection of voltage sag using unscented Kalman smoother," in Proc. International Conference on in Environment and Electrical Engineering (EEEIC), pp. 128-131, 2010 .

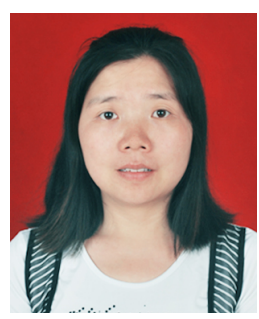

Yanhui Xi She received the B.S and M.S degrees in electrical and information engineering from Hunan Normal University, China, in 2002 and 2005, respectively. She earned the Ph.D. degree in Central South University, China, in 2013. Her research interests are traveling identification and signal

processing.

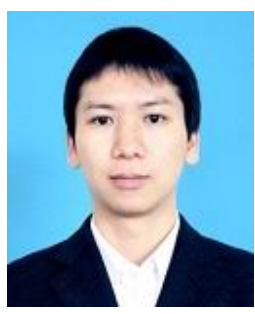

Zewen Li He received the B.S, M.S and $\mathrm{Ph} . \mathrm{D}$ degrees in electrical engineeering from Hunan University, China, in 1998, 2004 and 2011, respectively. His major interests include traveling wave fault location technology and wide-area traveling wave protection technology.

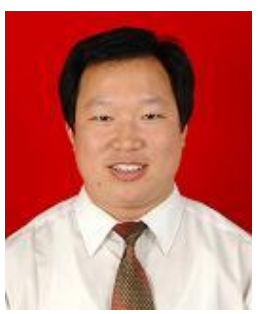

Xiangjun Zeng He received the B.S degree from Hunan University, China, in 1993. He earned the M.S and Ph.D degrees in electrical engineering from Huazhong University of Science \& Technology, China, in 1996 and 2001, respectively. His major interest is realtime application in power systems control and protection.

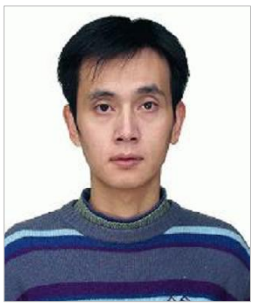

Xin Tang He received the M.S. and $\mathrm{Ph} . \mathrm{D}$. degrees in control engineering from Central South University, China, in 2001 and 2005, respectively. His research interests include repetitive control, power electronics and motor control, and DSP-base control system. 